Meta

Journal des traducteurs

Translators' Journal

\title{
La polyphonie textuelle balzacienne en espagnol et en anglais
}

\section{Pablo Zambrano Carballo}

Volume 57, numéro 3, septembre 2012

URI : https://id.erudit.org/iderudit/1017084ar

DOI : https://doi.org/10.7202/1017084ar

Aller au sommaire du numéro

\section{Éditeur(s)}

Les Presses de l’Université de Montréal

ISSN

0026-0452 (imprimé)

1492-1421 (numérique)

Découvrir la revue

Citer cet article

Zambrano Carballo, P. (2012). La polyphonie textuelle balzacienne en espagnol et en anglais. Meta, 57(3), 626-646. https://doi.org/10.7202/1017084ar

\section{Résumé de l'article}

Définie par Balzac lui-même comme « l'oeuvre capitale dans l'oeuvre ", Illusions perdues révèle la plupart les stratégies, thématiques, narratives et stylistiques mises en oeuvre dans La comédie humaine. L'une de ces stratégies est la reproduction fidèle d'une grande variété de discours oraux et écrits. Le présent article fait état, en premier lieu, d'une analyse de la relation entre le concept aristotélicien de la mimesis et la technique réaliste de la vraisemblance, que Balzac fait reposer en grande partie sur des bases linguistiques et métalinguistiques. Par ailleurs, grâce à une comparaison de diverses traductions espagnoles et anglaises d'Illusions perdues, l'article examine une sélection représentative des discours contenus dans le roman et souligne l'importance capitale, pour les traducteurs, d'être conscients de la variété des styles dans l'oeuvre balzacienne, afin de restituer dans les langues d'arrivée un effet de vraisemblance comparable à celui de l'original. 


\title{
La polyphonie textuelle balzacienne en espagnol et en anglais
}

\author{
PABLO ZAMBRANO CARBALLO \\ Universidad de Huelva, Huelva, Espagne \\ zambrano@uhu.es
}

\begin{abstract}
RÉSUMÉ
Définie par Balzac lui-même comme «l'œuvre capitale dans l'œuvre», Illusions perdues révèle la plupart les stratégies, thématiques, narratives et stylistiques mises en œuvre dans La comédie humaine. L'une de ces stratégies est la reproduction fidèle d'une grande variété de discours oraux et écrits. Le présent article fait état, en premier lieu, d'une analyse de la relation entre le concept aristotélicien de la mimesis et la technique réaliste de la vraisemblance, que Balzac fait reposer en grande partie sur des bases linguistiques et métalinguistiques. Par ailleurs, grâce à une comparaison de diverses traductions espagnoles et anglaises d'Illusions perdues, l'article examine une sélection représentative des discours contenus dans le roman et souligne l'importance capitale, pour les traducteurs, d'être conscients de la variété des styles dans l'œuvre balzacienne, afin de restituer dans les langues d'arrivée un effet de vraisemblance comparable à celui de l'original.
\end{abstract}

\begin{abstract}
Being the central novel of La comédie humaine, Illusions perdues displays most of Balzac's thematic, narrative and stylistic strategies. One of those strategies is the faithful reproduction of a great variety of oral and written discourses. This paper analyses the relationship between the Aristotelian concept of mimesis and the nineteenth-century literary technique of realistic verisimilitude, and the way in which language and metalanguage are used as two essential tools in Balzac's narrative. Through a comparison of examples taken from several translations of Illusions perdues in Spanish and English, the ultimate goal of this paper is to emphasize the importance for translators to be aware of the stylistic variety of Balzac's novel in order to create in the target languages a realistic effect as near as possible to the original one.
\end{abstract}

\section{MOTS-CLÉS/KEYWORDS}

réalisme, langue balzacienne, métalangage, vraisemblance realism, Balzac's language, metalanguage, verisimilitude

\section{Introduction}

Le roman Illusions perdues représente la pierre angulaire de La comédie humaine, projet littéraire fleuve et inachevé conçu par Balzac entre 1829 et 1848. Ce fut l'auteur lui-même qui, le déclarant «œuvre capitale dans l'œuvre ${ }^{1}$ », le plaça au cœur même de son immense roman de romans. D’un côté, il sert de lien entre les deux scènes principales qui composent «les études de mœurs» de la vie provinciale et parisienne; de l'autre, il appartient, avec Le père Goriot et Splendeur et misères des courtisanes, au "cycle Vautrin» hanté, précisément, par la présence de ce mystérieux personnage, l'une des créations géniales de Balzac. D’une certaine façon, Illusions perdues peut être considéré comme une Comédie humaine à moindre échelle, et dans cette perspective, 
on retrouve aisément bon nombre des axes thématiques, narratifs et stylistiques qui - du point de vue des présupposés réalistes - articulent l'ensemble.

Se fondant sur ce caractère exemplaire d'Illusions perdues, le présent article se propose d'analyser, par le biais d'une étude comparée de diverses traductions (voir les références en annexe), la langue balzacienne et les divers problèmes qui peuvent se poser dans sa transposition en espagnol et en anglais. En premier lieu, la nature pluri-stylistique et polyphonique du roman, ainsi que le souci linguistique et métalinguistique avoué sont liés au concept général de la mimesis aristotélicienne et à son application tant aux idéaux de l'esthétique réaliste du $\mathrm{XIX}^{\mathrm{e}}$ siècle qu'à son exigence de vraisemblance: l'un des axes fondamentaux de cette dernière passe précisément par la reproduction la plus fidèle possible de multiples types de discours oraux et écrits. Ce lien entre réalisme et langue nous mène à considérer la variété des styles que déploie Balzac dans Illusions perdues et qui fonde profondément le projet réaliste de l'auteur. Une fois posée l'importance capitale de la langue comme source de vraisemblance, l'article s'attache à souligner la nécessité de fixer un objectif fondamental au cours du processus traductologique: il est essentiel de restituer la nature pluristylistique qui caractérise le texte original afin de créer dans la langue d'arrivée, un effet de réalité que les lecteurs puissent décoder sans interférences majeures de sorte à égaler, dans la mesure du possible, celui proposé par Balzac.

\section{Réalisme et langue: Illusions perdues, un roman réaliste (méta)linguistique et polyphonique}

La relation complexe et insondable entre la réalité et sa représentation a dominé le débat artistique et littéraire depuis les formulations primitives platonicienne et aristotélicienne. S'il est un point que nous pouvons admettre avec une quasi-certitude, c'est que toute mimesis, en tant que représentation de la réalité, n'est qu'une illusion référentielle que le lecteur considère et qui mène nécessairement à distinguer le vrai - appartenant à l'ordre de la réalité - et le vraisemblable, en tant que concept artistique né de la poétique. En tant que concept poétique, la vraisemblance du discours réaliste - celui du XIX ${ }^{\mathrm{e}}$ siècle concrètement - se fonde sur un ensemble de stratégies de natures diverses qui, bien qu'encore non définies ni systématisées avec une totale précision par la critique, déterminent la perception du réel du lecteur qui reçoit et décode un discours particulier. Ainsi, Barthes, par exemple, perçoit l'écriture réaliste du XIX ${ }^{e}$ siècle comme un discours saturé de signes qui dénoncent sa fabrication illusoire; Rifaterre parle des références à la réalité comme d'une gymnastique verbale à laquelle le lecteur se voit contraint par le texte lui-même; Jakobson, tout en soulignant l'aspect fluctuant de cette notion, perçoit le réalisme comme une pure convention esthétique, quoique sans normes formelles fixes, mais indique malgré tout la métonymie et la synecdoque comme caractéristiques essentielles du discours réaliste du $\mathrm{XIX}^{\mathrm{e}}$ siècle; enfin, Auerbach relève, quant à lui, entre autres caractéristiques, le mélange de registres des styles employés. Ce sont toutes ces approches auxquelles nous renvoie Hamon (1973: 424-444), auteur de l'une des principales tentatives de systématisation des conventions du discours réaliste, qu'il qualifie graphiquement de «discours contraint». Des quinze marqueurs réalistes répertoriés par Hamon ${ }^{2}$, trois ou quatre au moins ont trait à des éléments qui partiellement ou totalement renvoient au plan linguistique: la motivation systématique des noms propres, des surnoms et 
des toponymes (marqueur 3), le travail linguistique de la dénomination (marqueurs 7 et 15) et le mimétisme discursif (marqueur 15) (Hamon 1973: 424-444). Préalablement, Hamon aborde la question inévitable des relations entre la réalité et sa mimesis littéraire, qu'il résume par le biais d'une question pertinente: «Peut-on reproduire par une médiation sémiologique (avec des signes) une immédiateté non sémiologique?» (Hamon 1973: 416). Autrement dit, quelles sont les limites auxquelles est confrontée la langue dans sa représentation de la réalité? Jusqu’à quel point la langue peut-elle être un outil fiable d'une représentation vraisemblable de la réalité ? Considérer que le degré de vraisemblance est proportionnellement déterminant pour la nature réaliste d'un texte, et par conséquent, pour le décodage réaliste ultime que le lecteur doit faire de lui-même, signifierait également que la mimesis purement textuelle - la linguistique - est l'une des plus aptes à contribuer à accroître le degré de vraisemblance de l'œuvre dans son ensemble. Partant de ce point, il serait même possible d'affirmer - tout en étant conscient des problèmes soulevés alors - que la mimesis textuelle participe même plus que la mimesis descriptive à la vraisemblance réaliste, puisque les mots passés dans le discours narratif sont ou pourraient être les mêmes que ceux de la réalité, tandis que les objets, lieux et personnages représentés par la description sont toujours distincts puisque fictifs (Déruelle et Rullier-Theuret 2003: 171). En allant plus loin encore, de telles considérations pourraient mener à l'extrême: d'un côté, considérer qu'« un énoncé linguistique ne peut alors "reproduire" qu'un énoncé ou un fragment d'énoncé linguistique identique déjà dit par lui-même [...] ou déjà dit par un autre énoncé linguistique» (Hamon 1973: 416); de l'autre, admettre l'idée que certains éléments de la réalité (sons, lignes, etc.) puissent être reproduits grâce à l'écriture: onomatopées, effets calligraphiques, etc. Dans le premier cas, Hamon parle de «réalisme textuel» et dans le second, de «réalisme symbolique» (Hamon 1973:416) ${ }^{3}$.

Indépendamment de la complexité sous-jacente à ce qui, de manière assez succincte, a été exposé, Balzac initie assurément, de même que Stendhal (Auerbach 1983: 441), un nouveau mode de vraisemblance réaliste dans lequel, précisément, la mimesis textuelle - à côté de la mimesis symbolique - occupe une position prééminente. C'est dans ce sens qu'Illusions perdues, véritable synthèse de l'ensemble de La comédie humaine, recèle une complexité qui se transcrit d'une manière évidente - et consciente chez l'auteur - sur les plans linguistique et stylistique. L'obtention de la vraisemblance, but ultime de la technique réaliste, conduit Balzac à prêter une attention minutieuse à la langue utilisée et à ses possibilités comme instrument de reproduction fiable de la réalité, ce qui débouche sur une conscience aiguë métalinguistique qui se manifeste en continu tant dans la voix du narrateur que dans celle de certains des personnages de l'œuvre. La technique réaliste de Balzac ne se pratique pas seulement dans la description de lieux, d'objets et de personnages; la reproduction de la réalité à laquelle aspire le réalisme est aussi celle du langage et des différents types de discours que cette réalité génère. Pour la mimesis textuelle dans Illusions perdues - et dans La comédie humaine en général - Balzac se sert de la reproduction d'une vaste palette de discours oraux qui se manifeste, comme nous allons le voir, par l'usage de termes idiomatiques, familiers, spécialisés chez les personnages et le narrateur omniscient lui-même. De plus, il reproduit différents types de discours écrits (lettres, articles de presse, recensions théâtrales, cartes de visite, affiches publicitaires, etc.), une caractéristique d'Illusions perdues qui la distingue ainsi des autres romans de La 
comédie humaine. Balzac utilise les péripéties de ses personnages, surtout de Lucien dans les éditoriaux, les journaux et le théâtre, pour inclure dans le roman les sociolectes et idiolectes les plus représentatifs de la société de l'époque, alors en pleine mutation. Illusions perdues se métamorphose ainsi en roman polyphonique au sein duquel cohabite un amalgame de discours qui incluent des lexiques spécialisés et des tournures empruntées à des sphères aussi diverses que la littérature, l'imprimerie, le journalisme, l'économie, le droit, la justice, l'agriculture ou la mode, entre autres. Le monde bigarré de La comédie humaine se traduit dans Illusions perdues par une véritable Babel polyphonique qui conduit parfois à l'incompréhension des personnages et celle du lecteur devant la langue utilisée, ce qui contraint le narrateur à de fréquentes interventions métalinguistiques dont le but est, d'une part, d'éclairer la signification spécifique de certains mots ou locutions afin de faciliter la communication et la compréhension et, d'autre part, de s'assurer que le lecteur respecte le pacte de vraisemblance. Devant cette polyphonie, il n'est pas étonnant que les puristes de l'époque aient accusé Balzac de rompre avec l'idéal linguistique de la littérature classique, qui se nourrissait de l'usage d'une langue intelligible par tous (Déruelle et Rullier-Theuret 2003: 175) et ne laissait pas de place à un répertoire lexical dont la diversité est un élément essentiel du nouveau mode de vraisemblance réaliste. Avec l'ouverture de la langue littéraire à la représentation d'une infinité de discours oraux - mais aussi écrits - de nature familière, technique, argotique, etc. qui auparavant n'avaient jamais joui d'un véritable statut littéraire, Balzac parvient à donner un tour de vis supplémentaire à la représentation vraisemblable de la réalité, qui est toujours polyphonique. Maintenir dans la traduction l'énorme effet réaliste produit par cette polyphonie constitue, à n'en pas douter, l'un des principaux défis auxquels doit faire face le traducteur qui se plonge dans Illusions perdues en particulier et dans La comédie humaine en général.

\section{La traduction de la polyphonie balzacienne dans Illusions perdues}

\subsection{La terminologie technique}

Malgré l'attention que Balzac prête à l'usage spécifique du lexique dans Illusions perdues, le narrateur omniscient de son roman recourt fréquemment, dans son aspect métalinguistique déjà souligné, au mot «argot» pour faire référence généralement à ce qui, de manière plus précise, pourrait être qualifié de diverses manières, selon la catégorie à laquelle renvoie le vocabulaire utilisé. Balzac lui-même, dans Des mots à la mode (1830) nomme "parlotte» ce que son narrateur appelle «argot». Quoi qu'il en soit, il apparaît qu'en réalité dans la terminologie de l'auteur se mêlent indifféremment diverses nuances qu'il convient d'éclairer. D'une part, Balzac recourt à ce que Damourette et Pichon (1971, cités dans Déruelle et Rullier-Theuret 2003 : 225) nomment disance, c'est-à-dire la langue utilisée par ceux qui exercent une profession déterminée ainsi que l'ensemble des termes techniques qui désignent l'activité, les outils et les produits d'une facette de l'activité humaine et sont le plus souvent incompréhensibles pour les personnes extérieures à ce groupe. Dans Illusions perdues, nombreux sont les termes appartenant au registre de la disance. En ce qui concerne le vocabulaire technique spécialisé, et donc hautement dénotatif, sa traduction présente les caractéristiques habituellement trouvées dans la traduction de n'importe quel discours technique non littéraire. 
La disance relative à l'imprimerie fournit une part significative des termes techniques et est essentiellement présente au début du roman, avec la description d'une vieille imprimerie de province et son fonctionnement, et dans la troisième partie, lorsque les expériences autour de la fabrication du papier acquièrent une importance fondamentale pour le dénouement de l'histoire. Une sélection de passages représentatifs permettra d'illustrer la présence mentionnée de termes typographiques spécialisés dans Illusions perdues et les problèmes spécifiques de traduction qu'ils présentent:

(1) Il s'y trouvait le billet de faire-part d'un mariage. Le vieil Ours abaissa la frisquette sur le tympan, le tympan sur le marbre qu'il fit rouler sous la presse; il tira le barreau, déroula la corde pour ramener le marbre, releva tympan et frisquette avec l'agilité qu'aurait mise un jeune Ours.

(Balzac 1837-1843/1977: 131)

(2) - Tu donnes le nom de têtes de clou aux Bâtardes, aux Coulées, aux Rondes de monsieur Gillé, anciennement imprimeur de l'Empereur! [...] Le vieux Séchard attrapa quelques cornets pleins de sortes qui n'avaient jamais servi et les montra.

(Balzac 1837-1843/1977: 132)

(3) La plus petite ramette, les ais, les jattes, la pierre et les brosses à laver, tout était chiffré avec le scrupule d'un avare [...] Tout en épiant la pensée de son fils [...], il amena successivement David devant une presse à satiner, une presse à rogner pour faire les ouvrages de ville, et il lui en vanta l'usage et la solidité.

(Balzac 1837-1843/1977: 133)

(4) À la fois compositeur, metteur en pages, et prote de l'imprimerie, Cérizet réalisait ce que Kant appelle une triplicité phénoménale.

(Balzac 1837-1843/1977: 563)

Bien qu'il s'agisse d'une langue technique qui, a priori, devrait faciliter une ample convergence dans les choix de traduction, les différentes versions présentent d'importantes discordances, fruit à plusieurs occasions d'un manque de rigueur dans la quête du terme exact dans les langues d'arrivée. En ce sens, il convient de souligner ici que, indépendamment du fait qu'il s'intègre dans un texte indiscutablement littéraire, le lexique d'une disance spécifique ne se défait jamais de la rigueur et du caractère éminemment révélateur qui caractérise les vocabulaires techniques. Leur incorporation dans Illusions perdues est une stratégie singulière de la technique réaliste de Balzac qui doit être interprétée avant tout dans une perspective littéraire ayant pour finalité l'obtention de la vraisemblance et le renouvellement de la langue littéraire à laquelle l'auteur aspire dans Illusions perdues et dans d'autres romans de La comédie humaine. Cela étant, la pertinence d'une telle analyse littéraire, qui aussi intéressante soit-elle, dépasse le cadre de cet article, n’annule aucunement la spécificité technique $\mathrm{du}$ vocabulaire en question ainsi que l'obligation, pour toute traduction, de restituer cette spécificité, comme ce serait le cas s'il s’agissait non pas d'un texte littéraire, mais technique.

Dans ses grandes lignes, la traduction des termes techniques présents dans les exemples de textes qui figurent ci-dessus ainsi que dans le reste du roman doit tenir compte de l'application de l'équivalence indispensable entre les deux langues concernées. Ainsi: 
1) un terme général et polysémique en français (par exemple pierre) peut être restitué par le biais d'équivalents généraux et polysémiques qui activent des signifiés spécifiques dans le contexte (piedra, stone);

2) à un terme français général et polysémique (brosse) peut correspondre un mot spécifique (en espagnol, bruza);

3) à un terme spécifique en français (par exemple frisquette) correspond un autre terme spécifique dans les langues d'arrivée (frasqueta, frisket);

4) le mot spécifique en français (prote) est restitué par le biais des termes polysémiques (regente, foreman).

Dans les textes qui nous intéressent, les termes polysémiques comme ais et jattes, qui dans l'original ont des signifiés spécifiques dans le contexte linguistique de l'imprimerie, sont restitués en espagnol, sans problème majeur, grâce à d'autres termes aux mêmes propriétés polysémiques (ainsi, en général, dans toutes les traductions espagnoles, planchas de madera ou tablas pour ais et cuencos pour jattes). Néanmoins, hormis Hunt (Balzac 1837-1843/2004: 13), qui traduit en 1971 pierre par stone et jattes par basins, les choix faits en anglais tendent à limiter sensiblement la polysémie originale en reportant l'unique spécification adjectivale de l'original français (brosses à laver) sur le reste des termes de la traduction (ainsi wetting-boards pour ais, pastepots pour jattes et rinsing trough pour pierre) et en éclairant dès lors aux yeux du lecteur anglophone le sens concret de certains ustensiles dont la finalité pratique demeure indéfinie pour les lecteurs espagnols et français ${ }^{4}$. La traduction de brosses en espagnol sert précisément à illustrer le second type d'équivalence mentionné (la polysémie traduite par le biais de la spécificité). La polysémie du terme français peut bien être restituée en espagnol grâce à un terme général, cepillos (Balzac 1837-1843/ 2007: 21, traduit par Monreal) - comme brushes dans les textes anglais -, ce qui débouche dans ce contexte sur le sens technique et spécifique propre à l'imprimerie. Cependant, en espagnol, l'option polysémique cepillos rivalise à son désavantage avec une autre, clairement spécifique dans le langage typographique, bruzas. Bruza est un gallicisme, précisément, de brosse qui couvre un champ sémantique spécialisé: " cepillo de cerdas muy espesas y fuertes, generalmente con una abrazadera de cuero para meter la mano, que sirve para limpiar las caballerías, los moldes de imprenta, etc. ${ }^{5}$ ", une option en faveur de laquelle se prononcent Cansino (Balzac 18371843/1967 : 449) comme Zambrano (Balzac 1837-1843/2008: 57). Dans ce cas, l'effet est opposé: par le biais de l'introduction d'un terme technique monosémique, le lecteur espagnol, qui n'a pas de raison de connaître la signification de bruzas, est privé de l'information d'une finalité pratique que l'original, lui, transmet. En réalité, bien que cepillos soit recevable et justifié comme traduction de brosses, en vertu de l'application de la première équivalence indiquée (la polysémie pour la polysémie), il est impossible de nier que la terminologie espagnole de la typographie offre un terme professionnel bruzas qui, dans sa spécificité, peut contribuer à l'effet de vraisemblance que Balzac poursuit dans son roman en introduisant un vocabulaire technique et, de plus, compenser d'autres moments où le recours aux équivalences implique irrémédiablement une réduction, fut-elle minimale, de la spécificité technique de l'original, comme cela arrive, par exemple, dans le cas de prote («A. - 1. Vieilli. Chef d'atelier dans une imprimerie. [...] 2. Chef du service de la composition dans une imprimerie typographique $\left.{ }^{6} . »\right)$ traduit par regente («3. m. En las imprentas, boticas, etc., hombre que sin ser el dueño dirige inmediatamente las operaciones $\left.{ }^{7} . »\right)$ et par foreman («4. a. the principal workman; spec., one who has charge of a department of work $\left.{ }^{8} »\right)$. 
En tous les cas, le choix de l'une ou l'autre des équivalences mentionnées précédemment, ainsi que son effet lors de la réception par le lecteur, devrait toujours tenir compte de la théorie générale de la compensation, ce qui est parfois soustrait du texte original resurgissant dans la mesure du possible en d'autres endroits.

\subsection{La terminologie sociolectale}

La reproduction des discours oraux prend une importance encore plus grande dans le cas de mimesis des sociolectes variés des différents groupes sociaux que Balzac évoque dans Illusions perdues. La notion de sociolecte renvoie à l'existence de certaines constantes linguistiques caractéristiques d'un niveau social déterminé au sein duquel les individus utilisent des vocables et des tournures dont l'usage est établi par les habitudes propres à cette classe. Les sociolectes permettent ainsi de percevoir, derrière les mots employés individuellement par les personnages, les discours sociaux dans lesquels ils s'intègrent. Leur analyse peut fournir des données tout à fait révélatrices des façons d'être, de penser et d'agir des groupes sociaux concernés, ainsi que de leur échelle de valeurs, de leurs qualités, de leurs défauts, etc. Il est clair que, dans Illusions perdues, les sociolectes les plus apparents et les plus intéressants sous l'angle de la traduction sont ceux des classes populaires, car leur lexique déteint en permanence sur celui de la bourgeoisie et même parfois, mais de façon beaucoup moins perceptible, sur celui de l'aristocratie.

Nous avons précédemment montré comment Balzac mise sur un style de langue littéraire qui rompt avec l'idéal linguistique de la littérature classique française. Pour preuve, comme nous l'avons vu, l'usage d'une vaste gamme de termes techniques intégrés dans le langage littéraire. Il convient d'ajouter à cela que Balzac écrit à une époque où le français populaire se fond peu à peu dans la langue écrite. Un tel transfert est évident dans Illusions perdues, tant dans le discours direct des personnages (même si cela est encore assez limité) que, surtout, dans le discours indirect du narrateur omniscient. Le rendu en d'autres langues de ce langage populaire, outil essentiel du réalisme balzacien, se révèle également fondamental pour que la traduction parvienne à créer un effet de réalité équivalent à celui qui figure dans le texte d'origine. Dans ce cas, comme dans beaucoup d'autres, les traducteurs sont de nouveau contraints à faire appel à des techniques de compensation (Baker 1992; Tricás Preckler 2003) qui permettent de réintroduire à d'autres endroits du roman les pertes qui se produisent lorsque l'on est dans l'impossibilité de rendre d'une façon appropriée en espagnol ou en anglais le caractère particulier d'une tournure donnée ou d'un terme populaire.

C'est ce qui se produit, par exemple, avec le terme saveteuse, que nous entendons dans la bouche d'une modiste anonyme qui arrive à la rédaction d'un journal:

(5) -...-$]$ Ainsi, tout est bien entendu: vous ne parlerez plus de Virginie, une saveteuse incapable d'inventer une forme, tandis que j'invente, moi!

(Balzac 1837-1843/1977: 332)

La complexité sémantique et morphologique de saveteuse rend ardue une traduction adéquate dans d'autres langues. Avant tout, il s'agit d'un mot qui n'est documenté dans aucun autre texte, qui, comme le note Chollet (Balzac 1837-1843/1977: 1252), n'est pas même répertorié par Dagneaud (1954) dans son analyse classique du 
lexique populaire de La comédie humaine, et que, pour leur part, Déruelle et RullierTheuret (2003: 226) présentent comme un néologisme balzacien. Quoi qu'il en soit, dans l'invention lexicale de Balzac convergent, d'une façon ou d'une autre et à un degré divers, quelques-uns des sens multiples de savate:

A. - 1. Chaussure, pantoufle usée [...] 3. P. anal. ou au fig., fam., péj. a) Personne maladroite ou incapable, imbécile, nullité [...] b) "Ouvrage mal fait, chose abîmée, gâchée" (Delvau 1883). [...] B. - [...] 2. Lutte à coups de pied en vogue surtout au XVIII siècle et au début du XIX ${ }^{\mathrm{e}}$ siècle [...] 3. Châtiment infligé autrefois à un soldat (ou, par extension, à un confrère de certaines corporations) par ses camarades qui, à tour de rôle, appliquaient sur les fesses dénudées de la victime un coup de soulier ferré9.

Trésor de la langue française informatisé (s.d.)

La dénommée Virginie, modiste elle aussi, est une saveteuse parce que:

a) elle est une personne inutile (deuxième acception de savate);

b) les chapeaux qu'elle crée sont de médiocre qualité (troisième acception renforcée par le lien entre saveteuse et savetier comme le pendant féminin de celui que le Trésor de la langue française informatisé (TLFi) définit ainsi: «A. - 1. Vx. artisan qui répare les chaussures [...] 2. P. anal., fam. Celui qui manque de créativité, d'originalité, de talent dans l'exercice de son métier, de son $\left.\operatorname{art}^{10} »\right)$;

c) c'est une femme des faubourgs, vulgaire et querelleuse, soit les quatrième et cinquième acceptions, renforcées par une autre acception populaire de savate, moins connue et non répertoriée, par exemple dans le TLFi, mais que Sainéan (1972) donne comme synonyme de chérie dans les milieux galants et de la prostitution.

Comme on peut l'observer, le néologisme de Balzac renvoie en français à un large ensemble de sens, plus ou moins populaires, voire vulgaires, impossibles à transposer totalement dans un seul terme espagnol ou anglais. Les traductions en ces langues tendent en général à se concentrer sur les deuxième et troisième acceptions du terme original, traduisant ainsi saveteuse par chapucera ( 1 . adj. Hecho tosca y groseramente. 2. adj. Dicho de una persona: Que trabaja de este modo ${ }^{11}$.») et bungler («a clumsy unskilful worker $\left.{ }^{12} »\right)$; ces choix présentent, à n'en pas douter, l'avantage de restituer grâce à un terme également familier (surtout en espagnol) une partie du signifié et du caractère populaires originels, mais renoncent irrémédiablement au reste des connotations, gommant en outre l'audace linguistique de Balzac en forgeant le néologisme. En dépit de la difficulté que présente la traduction du vocable original, la solution proposée par Marriage (Balzac 1837-1843/2009: 214), botcher, mérite particulièrement d'être considérée, parce qu'elle réussit partiellement la tâche ardue d'embrasser au plus près possible l'ample polysémie de saveteuse. En effet, botcher s'avère une traduction à l'évidence plus pertinente parce que:

a) le mot remplit d'entrée de jeu le champ sémantique couvert également par bungler (dont il est le synonyme) et par le terme espagnol chapucera (acceptions françaises $3 a$ et $\left.3 b^{13}\right)$;

b) il est le synonyme de cobbler, dont diverses acceptions reprennent la première de saveteuse ("A. - 1. chaussure, pantoufle usée $\left.e^{14} »\right):$ : 1 a. One whose business it is to mend shoes [...] 2. One who mends clumsily, a clumsy workman, a mere botcher ${ }^{15}$ ";

c) comme synonyme de «2. [...] b. a tailor who does repairs ${ }^{16} »$, il indique aussi indirectement une activité, celle de la modiste gauche, en lien avec celui de la dénommée Virginie; 
d) comme synonyme aussi de jobber («4. a. a person who uses a public office or position of trust for personal gain or political advantage $\left.{ }^{17} »\right)$, et lié par conséquent à jobbery ( 1 1. the practise of turning a public office or position of trust to personal gain or political advantage; the execution of corrupt jobs $\left.{ }^{18} »\right)$, botcher finit même par inclure, indirectement, l'intention malveillante qui anime la modiste anonyme de Balzac à discréditer une rivale.

Le vocabulaire de la presse se révèle des plus productifs dans la seconde partie d'Illusions perdues. D’un côté, il apporte, comme celui de l'imprimerie, une importante quantité de termes techniques propres à sa disance et de l'autre, il se présente comme l'un des sociolectes les plus représentatifs et identifiables du roman. Celui-ci est en effet caractérisé autant par une forte influence de termes familiers et vulgaires que par l'argot particulier des journalistes, incompréhensible pour les non-initiés, au point que Lucien lui-même, déconcerté, a besoin d'éclaircissements métalinguistiques à plusieurs reprises ${ }^{19}$.

Le mot rossignol servira à illustrer partiellement ce sociolecte ainsi que l'imbrication stylistique subtile que Balzac réalise entre le terme choisi et certains jeux de mots disséminés dans l'œuvre, imbrication que la traduction doit tenter de restituer, au moins en partie.

(6) - J'ai fait deux cents Petit vieillard de Calais; mais il a fallu, pour les placer, déprécier deux autres ouvrages sur lesquels on ne nous faisait pas de si fortes remises, et qui sont devenus de fort jolis rossignols.

Plus tard Lucien apprit que ce sobriquet de rossignol était donné par les libraires aux ouvrages qui restent perchés sur les casiers dans les profondes solitudes de leurs magasins.

(Balzac 1837-1843/1977: 302)

Dans ce cas, c'est le narrateur lui-même - ou en d'autres occasions l'un des personnages - qui définit le mot que Lucien, en principe, ne comprend pas. La définition du narrateur coïncide avec l'une des acceptions consignées, par exemple, par le TLFi: «C. Fam., gén. iron. ou péj. 1. a) Livre sans valeur, qui date, qui reste longtemps sur les rayons de la librairie [...] b) marchandise démodée, défraîchie, difficilement vendable ${ }^{20} »$. Le narrateur, cependant, suggère un commentaire étymologique ironique en liant métaphoriquement et subtilement les œuvres qui demeurent invendues sur les rayons des librairies avec des oiseaux posés ("perchés») sur les branches d'un $\operatorname{arbre}^{21}$, une métaphore filée déjà quelques pages plus loin:

(7) Ces élégants rossignols, vendus un peu plus cher que les autres à cause de leur papier vélin, viennent presque tous s'abattre sur les rives de la Seine, où vous pouvez aller étudier leurs chants, si vous voulez faire un jour quelque pèlerinage instructif sur les quais de Paris, depuis l'étalage du père Jérôme, au pont NotreDame, jusqu'au Pont-Royal.

(Balzac 1837-1843/1977: 341-342)

Le commentaire métalinguistique du narrateur et l'identification métaphorique de livres et d'oiseaux rendent possible, et souhaitable, une traduction littérale (ruiseñores / nightingales) adoptée par tous les traducteurs, hormis Monreal (Balzac 1837-1847/2007a $)^{22}$. La traduction littérale est conseillée, voire nécessaire, parce que rossignol n'est autre que le chaînon d'un vaste et ironique jeu de mots pour lequel Balzac s'appuie sur deux autres acceptions du terme français: «2. a) Personne qui se 
distingue par une voix bien modulée, qui chante admirablement [...] b) Poète lyrique, écrivain au talent prestigieux ${ }^{23} »$. En effet, bien avant dans le roman, le narrateur se fait l'écho d'une occurrence linguistique chez un personnage mineur qui se réfère à Lucien comme au «chardonneret du sacré boccage» (Balzac 1837-1843/1977: 172), un jeu de mots autour du nom de l'oiseau en question (jilguero en espagnol, goldfinch en anglais) et du nom du protagoniste (Chardon) ${ }^{24}$. Évidemment, la subtilité du jeu linguistique original se perd dans les traductions, bien que cela puisse s'expliquer en note, comme le font Monreal, Zambrano et Hunt, mais tous maintiennent au moins le lien entre un écrivaillon comparé à un chardonneret (jilguero, goldfinch) et son œuvre, invariablement condamnée à devenir un rossignol (ruiseñor, nightingale).

À ce stade, il convient de souligner l'avantage de l'anglais par rapport à l'espagnol lorsqu'il s'agit de traduire avec la plus grande fidélité possible et par le biais de techniques compensatoires, le jeu de mot original. D'une part, bien que les principaux dictionnaires d'espagnol ne relèvent aucun sens figuré du terme, il n'en est pas moins certain que la traduction littérale de rossignol dans cette langue fonctionne parce que, indépendamment des dictionnaires, les locuteurs établissent sans grande difficulté le sens figuré de ruiseñor comme «chanteur à la voix mélodieuse». Néanmoins, le terme anglais nightingale est plus pertinent étant donné qu'il contient non seulement le sens figuré mentionné ( 2 . a person with a sweet or enchanting voice; one who sings or speaks delightfully $\left.{ }^{25} »\right)$, mais qu'il ajoute également une composante humoristique («1. [...] c. with distinguishing word: an animal, esp. a frog, with a loud, unmusical call $\left.{ }^{26} »\right)$ qui vient compenser dans la traduction l'ironie balzacienne jouant avec l'acception déjà citée de rossignol comme "poète lyrique, écrivain au talent prestigieux", c'est-à-dire précisément l'opposé de ce que nombre d'autres personnages du roman pensent de Lucien. La plus grande richesse connotative de l'anglais nightingale pousse, consciemment ou non, quelques traducteurs (Balzac 1837-1843/ 2001 : 57, traduit par Raine 1951; Balzac 1837-1843/2009: 48, traduit par Marriage) à traduire chardonneret non par goldfinch, mais par songster, un terme au champ sémantique plus vaste et qui jouit de l'avantage inégalable de réunir les trois sens, réels et figurés, avec lesquels Balzac joue en français: «1. a. One who sings, a singer [...] 2. A poet; a writer of songs or verse 3. A bird that sings; a song-bird ${ }^{27} »$. De sorte que, d'une certaine façon, la perte irrémédiable de nuances entraînée par la traduction est de nouveau compensée ${ }^{28}$.

La métaphore ornithologique autour de Lucien et les livres culmine, bien plus avant dans le livre, avec un troisième calembour:

(8) Cette défection fut le signal d'un effroyable tapage dans les journaux libéraux, Lucien devint leur bête noire, et fut tympanisé de la plus cruelle façon: on raconta les infortunes de ses sonnets, on apprit au public que Dauriat aimait mieux perdre mille écus que de les imprimer, on l'appela le poète sans sonnets!

(Balzac 1837-1843/1977: 516)

Le jeu fondé sur l'homophonie de sans sonnets et sansonnet (estornino / starling) est manifestement intraduisible et les options sont variées. Cansino (Balzac 18371843/1967: 691) choisit une voie intéressante: ne pas traduire le significatif écrit (sans sonnets), mais l'une des deux options se situant sur le plan phonétique, el poeta estornino (sansonnet), et éclairer le calembour dans une note en bas de page. Ce choix, s'il peut sembler étrange à première vue, a l'avantage de reprendre en espagnol les références à l'ornithologie de l'original. Zambrano, quant à lui (Balzac 1837-1843/2008: 
437), opte pour une traduction, el sonetista del sonsonete, qui fait abstraction de l'allusion ornithologique, rompt la continuité métaphorique de l'original, mais qui, en revanche, tente de restituer une partie du jeu phonétique et de l'ironie du narrateur grâce au terme sonsonete ( ( $2 . m$. ruido generalmente poco intenso pero continuado, y por lo común desapacible $\left.{ }^{29} »\right)$ qui fait état des vers de mirliton de Lucien. Hunt emprunte la même piste avec sonnetless sonneteer (Balzac 1837-1843/2004: 437, traduit par Hunt 1971), une proposition qui est une tentative de restitution à la fois phonétique et sémantique de l'original, puisque sonneteer n'est pas seulement, de manière neutre, «a composer of sonnets ${ }^{30} »$; il se réfère fréquemment à: «in disparaging sense, a minor or indifferent poet ${ }^{31} »$. Enfin, le renoncement aux restitutions phonétiques et sémantiques compensatoires du calembour original explique les traductions plus ou moins littérales comme the poet without sonnets (Balzac 18371843/2001: 448, traduit par Raine 1951), the poet sans poems (Balzac 18371843/2007b : 407, traduit par Prescott 1893) et the poet sans sonnets (Balzac 1837-1843/2009: 409, traduit par Marriage).

\subsection{La terminologie idiolectale}

L'un des outils fondamentaux du réalisme balzacien est, à n'en pas douter, la caractérisation des personnages qui, outre leur importance dans le roman et la classe ou groupe social auxquels ils appartiennent, sont dépeints dans la majorité des cas par un ou divers traits individuels qui les distinguent et les singularisent: le linge qu'ils portent, la maison dans laquelle ils habitent, leur lieu de travail, l'expression de leur visage ou, pour ce qui nous intéresse, leur mode particulier d'expression. Ainsi, un nombre important de personnages d'Illusions perdues et de La comédie humaine demeurent gravés certes dans la mémoire des lecteurs, mais plus particulièrement, de façon très singulière, dans leur mémoire auditive. $\mathrm{Si}$, comme nous l'avons vu, les termes techniques caractérisent linguistiquement l'individu en tant que membre d'un corps de métier et si les sociolectes l'incluent au sein d'un groupe social, politique ou autre, les idiolectes, c'est-à-dire les expressions qui lui sont propres, correspondent à une particularité unique et personnelle de l'individu: sa façon de s'exprimer. Comme dans les exemples précédents, le traducteur doit être à l'affût des nombreux idiolectes que Balzac introduit dans le roman afin de parvenir à une restitution correcte, avec toujours le même objectif qui est d'offrir au lecteur un reflet le plus fidèle possible de la nature polyphonique du texte d'origine et des diverses modulations linguistiques présentes dans les différents types de discours.

Dans la première partie d'Illusions perdues, Madame de Bargeton, l'un des principaux personnages du roman, est caractérisée par certains traits bovarystes avant la lettre qui nous la présentent comme une aristocrate s'ennuyant au sein d'une société provinciale étriquée et, par conséquent, plongée dans une rêverie romanesque dont elle se débarrassera lors de son arrivée à Paris, au cours de la seconde partie du roman. Entre-temps, et pour compenser l'ennui dans lequel elle est plongée à Angoulême,

(9) [e]lle palpitait, elle se pâmait, elle s'enthousiasmait pour tout événement: pour le dévouement d'une sœur grise et l'exécution des frères Faucher, pour l'Ipsiboé de monsieur d'Arlincourt comme pour l'Anaconda de Lewis, pour l'évasion de Lavalette comme pour une de ses amies qui avait mis des voleurs en fuite en faisant la grosse voix. Pour elle, tout était sublime, extraordinaire, étrange, divin, merveilleux. Elle s'animait, se courrouçait, s'abattait sur elle-même, s'élançait, retombait, 
regardait le ciel ou la terre; ses yeux se remplissaient de larmes. Elle usait sa vie en de perpétuelles admirations et se consumait en d'étranges dédains. Elle concevait le pacha de Janina, elle aurait voulu lutter avec lui dans son sérail, et trouvait quelque chose de grand à être cousue dans un sac et jetée à l'eau. Elle enviait lady Esther Stanhope, ce bas-bleu du désert. Il lui prenait envie de se faire sœur de Sainte-Camille et d'aller mourir de la fièvre jaune à Barcelone en soignant les malades: c'était là une grande, une noble destinée! Enfin, elle avait soif de tout ce qui n'était pas l'eau claire de sa vie, cachée entre les herbes. Elle adorait lord Byron, Jean-Jacques Rousseau, toutes les existences poétiques et dramatiques. Elle avait des larmes pour tous les malheurs et des fanfares pour toutes les victoires. Elle sympathisait avec Napoléon vaincu, elle sympathisait avec Méhémet-Ali massacrant les tyrans de l'Égypte. Enfin, elle revêtait les gens de génie d'une auréole, et croyait qu'ils vivaient de parfums et de lumière.

(Balzac 1837-1843/1977: 157-158)

Les élans exagérés de l'état d'esprit de Madame de Bargeton se répercutent sur son langage. Comme nous l'indique le narrateur,

(10) [e]lle avait le défaut d'employer de ces immenses phrases bardées de mots emphatiques, si ingénieusement nommées des tartines dans l'argot du journalisme qui tous les matins en taille à ses abonnés de fort peu digérables, et que néanmoins ils avalent. Elle prodiguait démesurément des superlatifs qui chargeaient sa conversation où les moindres choses prenaient des proportions gigantesques. Dès cette époque elle commençait à tout typiser, individualiser, synthétiser, dramatiser, supérioriser, analyser, poétiser, prosaïser, colossifier, angéliser, néologiser et tragiquer; car il faut violer pour un moment la langue, afin de peindre des travers nouveaux que partagent quelques femmes.

(Balzac 1837-1843/1977: 157; souligné par l’auteur)

Ainsi, son idiolecte la caractérise autant que son comportement ou sa façon de s'habiller. Il s'agit d'une langue parasite qui se nourrit en grande partie du lexique et de l'éloquence d'œuvres comme Ipsiboé du vicomte d'Arlincourt ${ }^{32}$, Anaconda, conte gothique de l'écrivain britannique Matthew G. Lewis ${ }^{33}$, et surtout Corinne ou l'Italie de Madame de Staël ${ }^{34}$, dont le vocabulaire et le style alimentent l'un des éléments caractéristiques de l'idiolecte de Madame de Bargeton, les tartines:

(11) De ses blanches mains, elle lui montra la gloire achetée par de continuels supplices, elle lui parla du bûcher des martyrs à traverser, elle lui beurra ses plus belles tartines et les panacha de ses plus pompeuses expressions. Ce fut une contrefaçon des improvisations qui déparent le roman de Corinne.

(Balzac 1837-1843/1977: 173; souligné par l'auteur)

Le terme tartine, issu de l'argot de la presse et du théâtre, apparaît au début $\mathrm{du} \mathrm{XIX}^{\mathrm{e}}$ siècle pour désigner un article de grandes dimensions ou un très long discours. La dégradation du langage de la presse que Balzac dénonce dans Illusions perdues est bien illustrée par ce terme, qui en constitue un exemple des plus pertinents. Saisir la métaphore culinaire est essentiel pour comprendre l'ironie qui se cache derrière ce vocable, car, comme Madame de Bargeton, Blondet ou Herrera le montrent dans chacune des trois parties du roman, la composition d'une tartine consiste précisément en l'application rigoureuse et mécanique d'une recette d'écriture (Déruelle et Rullier-Theuret 2003: 179). L'association des deux sens principaux de ce terme, «A. - 1. Tranche de pain recouverte d'une substance alimentaire qui s'étale facilement ${ }^{35}$ » et «B. - 1. Fam. Long développement écrit, long discours, souvent sans 
intérêt $t^{36} »$, explique l'usage de tailler (exemple 9). En effet, ce verbe a des acceptions distinctes et apparaît dans différentes expressions métaphoriques qui, avec une fréquence variable, surgissent dans le roman, dans un contexte global qui constitue précisément une satire du monde de la presse et de la manipulation linguistique exercée par ce dernier. On observe donc:

a) la signification courante, non métaphorique, de «I. - couper, trancher ${ }^{37}$ », que le TLFi illustre justement avec l'exemple: «A. - [...] tailler des tartines ${ }^{38} »$, tartine signifiant ici «tranche de pain»;

b) l'expression, tombée en désuétude, tailler les morceaux: «1. Locutions [...] b) Vieilli. Tailler les morceaux (à qqn) "Lui couper le pain, la viande en morceaux, de manière à ce qu'il n'ait plus qu'à manger" 39 ";

c) un sens associé au journalisme ou à l'édition de textes: «II - 2. [...] b) [Le suj. désigne un écrivain, un rédacteur de revue] Effectuer des coupures, supprimer des passages dans un article, un ouvrage, pour le réduire à l'essentiel ou pour le censurer. [...] Et le lendemain, je me suis remis à tailler dans ce gros roman dont chaque phrase m'a donné tant de mal (GREEN, Journal, 1931, p. 75) ${ }^{40}$.» Tailler dans un texte correspond exactement au contraire de tailler une tartine, dans le sens métaphorique;

d) l'expression familière tailler une bavette: «C. Fam. Tailler des bavettes. "Passer son temps en bavarderies, en commérages, en caquets" ${ }^{\prime 1}$ ». Cette expression donne lieu au jeu de mots de Jules Renard dans son Journal (1894), cité dans le TLFi à l'entrée bavette: «il cousait de plaisantes bavettes sous le nez des directeurs de journaux ${ }^{42}$ » où coudre signifie "rédiger». Bouclant la boucle, ce jeu de mots nous renvoie curieusement au monde du journalisme où est née l'acception familière de tartine, dans le sens d'un discours long, creux et ennuyeux.

Les traductions de tartine, terme argotique qui désigne un trait singulier du parler particulier de Madame de Bargeton, entre autres, doivent essayer, bien sûr, de restituer dans la mesure du possible l'ensemble des connotations. La solution, bien entendu, ne passe pas, comme le fait Prescott (Balzac 1837-1843/2007b: 36), par la suppression dans la langue cible du terme et du commentaire ironique qui l'accompagne. De nouveau, les traductions anglaises présentent l'avantage que l'emprunt français tartine, qui est choisi par le reste des traducteurs, est déjà documenté en 1804 dans son sens premier («a. a slice of bread spread with butter or preserve ${ }^{43} »$ ), ainsi que dans son sens figuré («b. fig. a big article of commonplace character $\left.{ }^{44} »\right)$ en 1907. Cependant, les dictionnaires d'usage les plus courants n'attestent même pas le mot tartine, ce qui explique peut-être que Hunt ait pris la décision discutable d'intervenir métalinguistiquement dans le texte cible et d'ajouter au commentaire ironique du narrateur une information purement lexicographique entre parenthèses: «tartines (slices of bread and butter)» (Balzac 1837-1843/2004: 40, traduit par Hunt 1971). Bien que cela soit discutable, il ne fait aucun doute qu'une telle information est destinée à faciliter la compréhension, par le lecteur, de la métaphore qui accompagne le terme pour le définir, métaphore qui, en anglais, est rendue par tous les traducteurs au moyen de vocables ayant une origine culinaire évidente: swallow, serve up, indigestible, etc. En ce qui concerne les traductions espagnoles, ces dernières restituent aussi sans difficulté tant les termes culinaires de la métaphore (tragar, servir, indigestos, atiborrar, ración, etc.) que le mot tartine lui-même, puisque la langue espagnole permet des traductions rendant compte des principales connotations du terme d'origine: tostada (Balzac 1837-1843/1967: 464, traduit par Cansino) et tostón (Balzac 1837-1843/2008: 89, traduit par Zambrano) possèdent, d'une part, un sens culinaire 
lié directement à celui de tartine ( $" 5$. $f$. Rebanada de pan que, después de tostada, se unta por lo común con manteca, miel u otra $\cos ^{45}{ }^{45}$ et « 2 . $m$. Rebanada de pan tostado empapado en aceite nuevo, que se unta con ajo y se adereza con sal o azúcar y zumo de naranja $\left.{ }^{46} »\right)$ et, d'une autre, un sens dérivé ( 7 . $m$. tabarra, lata $\left.{ }^{47} »\right)$ qui renvoie à l'idée de quelque chose ou de quelqu'un d'ennuyeux, de bavard et d'inconsistant.

Les tartines de Madame de Bargeton sont caractérisées, comme le remarque le narrateur d'Illusions perdues, par la nature emphatique et superlative des expressions et des termes qui les composent. Le commentaire ironique du narrateur le conduit à justifier le détournement des règles linguistiques afin de pouvoir reproduire avec exactitude, c'est-à-dire, avec réalisme, l'idiosyncrasie d'un idiolecte, celui de Madame de Bargeton, qui, de par son caractère exagéré, est commun à certaines femmes répondant au modèle de l'aristocrate d'Angoulême, vouée à l'ennui et vivant dans un songe romanesque permanent. Le détournement de la langue que le narrateur reproduit apparaît dans toute une série de termes, certains corrects, d'autres étranges ou inventés (typiser, colossifier, tragiquer, néologiser, etc.) qui, sans grande difficulté, sont restitués avec un effet semblable tant en espagnol (tipificar, colosificar, tragicar, neogilizar) qu'en anglais (tipify, colossify, tragedify, neologize).

L'invention ou le mauvais usage de vocables n'est pas absolument propre aux tartines qui caractérisent l'idiolecte de Madame de Bargeton. En fait, Balzac parsème son roman de toute une série de mots inventés ou corrompus dans la bouche d'un certain nombre de personnages ${ }^{48}$, y compris le narrateur omniscient lui-même, comme on le voit dans les exemples qui suivent.

La personnalité complexe de Lucien découle en grande partie de son désir de réussir dans le monde de la littérature et du journalisme et des oscillations entre un provincialisme dont il ne parvient pas à se débarrasser et un snobisme grâce auquel il prétend s'intégrer à l'élite parisienne. Cela le fait passer linguistiquement d'une langue régionale et provinciale (comme on le voit dans le terme restaurat qu'il inclut dans une lettre à sa sœur Ève) à des néologismes savants comme celui qu'il utilise dans sa réponse à la description que Losteau lui fait du critique littéraire Félicien Vernou:

(12) [...] As-tu maintenant envie de te marier? Vernou n'a plus de cœur, le fiel a tout envahi. Aussi est-ce le journaliste par excellence, un tigre à deux mains qui déchire tout, comme si ses plumes avaient la rage.

-Il est gunophobe, dit Lucien. A-t-il du talent?

(Balzac 1837-1843/1977: 427)

Gunophobe est une invention que Balzac met dans la bouche de Lucien pour justifier le caractère aigri et impitoyable de Vernou et de ses critiques littéraires attribué à l'influence pernicieuse de sa terrible épouse. Le sens de gunophobe est apparenté sans nul doute à celui de misogyne (les affixes guno- et -gyne ayant la même étymologie). Ce néologisme de Balzac, qui, contrairement à d'autres qu'il a forgés, n'a connu aucun succès, s'explique peut-être non seulement par la volonté de mettre dans la bouche de Lucien un terme en accord avec le snobisme du personnage, mais aussi parce que le mot misogyne, bien qu'attesté en français depuis 1564, est très peu utilisé jusqu'au XIX ${ }^{\mathrm{e}}$ siècle ${ }^{49}$. Dans le contexte linguistique de l'époque de Balzac, l'étrangeté du néologisme gunophobe était semblable à celle du terme misogyne. Un tel phénomène se produit aussi en anglais, puisque misogynist et misogyny, déjà documentés respectivement en 1620 et 1656, s'emploient beaucoup plus à partir du XIX ${ }^{\mathrm{e}}$ siècle. Le 
cas de l'espagnol est encore plus intéressant puisque le conservatisme linguistique du dictionnaire de référence, le Diccionario de la Real Academia Española, n’inclut les termes misógino et misoginia que très tardivement, 1925 et 1936 respectivement. Quoi qu'il en soit, ce qui est certain, c'est que Lucien, qui aurait pu utiliser misogyne, traite Vernou de gunophobe et il semble donc légitime que les traductions puissent donner la possibilité au lecteur d'apprécier l'audace linguistique de Balzac. Dans ce sens, toutes les traductions espagnoles restituent ce néologisme au moyen d'équivalents qui produisent un effet presque identique à celui du terme original et qui n'atténuent point la façon de s'exprimer du personnage: gunófobo (Balzac 1837-1843/1967: 637, traduit par Cansino), ginéfobo (Balzac 1837-1843/2007a: 359, traduit par Monreal) et ginófobo (Balzac 1837-1843/2008: 446, traduit par Zambrano). Ces deux derniers exemples sont accompagnés de notes en bas de page par lesquelles les traducteurs signalent le sens et le fait qu'il s'agit de néologismes. Cependant, en anglais, hormis la traduction de Marriage it is a case of gunophobia (Balzac 1837-1843/2009: 315), les traducteurs éliminent l'originalité du terme balzacien et le remplacent par le synonyme misogynist utilisé actuellement (Balzac 1837-1843/2004: 338, traduit par Hunt 1971), par une périphrase telle que woman-hater (Balzac 1837-1843/2001 : 345, traduit par Raine 1951) ou par une traduction partielle telle que he is a destructive (Balzac 1837-1843/2007b : 314, traduit par Prescott 1893), laquelle limite le sens original; des options qui s'éloignent de plus en plus de l'idiosyncrasie non seulement sémantique, mais aussi morphologique de gunophobe.

Le narrateur omniscient lui-même est caractérisé par certains traits de langue qui lui sont propres et qui contiennent aussi la création d'un nombre limité, mais original de néologismes. Ces derniers restituent au lecteur sa vision, condescendante ou parfois critique et souvent ironique aussi, des personnages du roman. C'est ce qui se produit dans le passage suivant:

(13) Montriveau présenta le baron du Châtelet à la marquise, et la marquise fit à l'ancien secrétaire des commandements de l'Altesse impériale un accueil d'autant plus flatteur, qu'elle l'avait déjà vu bien reçu dans les trois loges, que Mme de Sérizy n’admettait que des gens bien posés, et qu'enfin il était le compagnon de Montriveau. Ce dernier titre avait une si grande valeur, que Mme de Bargeton put remarquer dans le ton, dans les regards et dans les manières des quatre personnages, qu'ils reconnaissaient du Châtelet pour un des leurs sans discussion. La conduite sultanesque tenue par Châtelet en province fut tout à coup expliquée à Naïs.

(Balzac 1837-1843/1977: 279)

Le néologisme sultanesque, que le TLFi atteste pour la première fois en 1836 dans Le Lys dans la vallée ( Rare. Qui présente des caractéristiques propres au sultan; qui évoque sa cour, son harem ${ }^{50} »$ ), est utilisé par Balzac afin de poser un regard cruel et ironique sur un personnage, Sixte du Châtelet, qui, par son snobisme prétentieux, s'attribue non seulement la particule $d u$ pour anoblir son nom de famille, mais aussi pour jouer un rôle de don Juan à l'ancienne au sein de l'aristocratie figée d'Angoulême. Dans ce sens, l'image exotique du sultan dans son harem évoquée par le terme sultanesque se superpose avec cruauté à celle d'un Casanova fané, ce qui justifie ici pleinement la pertinence du néologisme. Comme dans le cas de gunophobe, l'espagnol adapte sans aucune difficulté et avec un rendu semblable le néologisme à la forme sultanesca, ce qui apparaît dans toutes les traductions. Toutefois, bien que l'Oxford English Dictionary (OED) atteste déjà le mot sultanesque en 1862 chez 
G. A. Lawrence («characteristic of a sultan $\left.{ }^{51} »\right)$, seul Raine (Balzac 1837-1843/2001 : 179, traduit par Raine 1951) l'adopte, tandis que le reste des traducteurs préfère remplacer le néologisme par des périphrases comme Châtelet's oriental affectations (Balzac 1837-1843/2004: 176, traduit par Hunt 1917) ou the dictatorial bearing of Du Châtelet (Balzac 1837-1843/2007b: 164, traduit par Prescott 1893), dont les effets sont beaucoup plus atténués.

Dans la troisième partie d'Illusions perdues, afin de parvenir à un réalisme vraisemblable, Balzac est conduit à souligner l'idiolecte de Kolb, le fidèle collaborateur de David, non pas au moyen de termes particuliers, comme dans les cas précédents, mais par le biais d'une transcription phonétique singulière - que l'auteur signale par l'italique - du fort accent alsacien du personnage, particularité qui rend parfois incompréhensible le message ${ }^{52}$ :

(14) On m'ovrirait pien tes millions, queu cheu ne tirais bas une motte! Est-ce che nei gonnais boind la gonzigne milidaire? (...) chesbère edre assez riche ein chour pire lui domper sire le gazaquin, à ced ôme te chistice! Ch'aime bas sa visache!

(Balzac 1837-1843/1977: 608)

Le défi du traducteur devant cet idiolecte n'est pas tant la restitution sémantique du discours, qui n'offre aucune difficulté réelle, mais son adaptation phonétique, car l'original exige que les lecteurs des traductions perçoivent clairement un fort accent allemand dans les paroles de Kolb, étant donné que cet accent est une idiosyncrasie de ce personnage balzacien. Bien entendu, la solution ne peut pas consister à supprimer ce trait essentiel de l'idiolecte de Kolb et à traduire ce qu'il dit par une langue cible plus ou moins standardisée, comme le font, par exemple, Zambrano Barragán et García (Balzac 1837-1843/1960: 706), Acerete (Balzac 1837-1843/1968: 457-458), Godó (Balzac 1837-1843/1964: 386) et Prescott (Balzac 1837-1843/2007b: 501), car cela conduit à réaliser un doublage inacceptable de son discours, ce qui ôte au personnage l'un de ses traits les plus caractéristiques. Dans les autres traductions espagnoles et anglaises, la solution consiste à recréer l'idiolecte de l'Alsacien au moyen de diverses stratégies fondées sur des modifications et des déformations phonétiques que le lecteur associera facilement à un discours espagnol ou anglais stéréotypé prononcé avec un fort accent allemand: dans le cas espagnol, tirría, tarle, pastante, poca, ofreciegan ou espego au lieu de respectivement diría, darle, bastante, boca, ofrecieran et espero; et en anglais, dey, opey, traw ou vass au lieu de they, obey, draw et was. Grâce à de telles stratégies, on obtient des traductions qui, au-delà de la perception du lecteur, présentent l'avantage de conserver dans les langues d'arrivée un trait propre à Kolb: son accent.

\section{Conclusions}

L'analyse que nous avons réalisée mène à la formulation de plusieurs conclusions importantes.

Tout d'abord, la technique réaliste de Balzac dans La comédie humaine en général et dans Illusions perdues, en tant que texte essentiel et servant de modèle pour l'ensemble, repose surtout sur la reproduction fidèle des discours oraux qui, grosso modo, peuvent être classés en trois grandes catégories: disances, sociolectes et idiolectes. L'importance que Balzac accorde à la vraisemblance linguistique apparaît chez 
le narrateur omniscient et chez beaucoup de ses personnages, qui truffent la narration de mots techniques, d'argot populaire et de parlers spécifiques, ce qui débouche souvent sur une incompréhension du protagoniste et des lecteurs eux-mêmes et conduit à de fréquentes interventions métalinguistiques du narrateur et des personnages. De plus, les sociolectes et les idiolectes permettent de caractériser les individus et les groupes sociaux auxquels ils appartiennent. C'est ainsi que l'analyse du lexique de la presse permet d'affirmer qu'il s'agit d'un vocabulaire qui montre à quel point, aux yeux de Balzac, le monde du journalisme pouvait être mal intentionné. De la même façon, l'étude de l'idiolecte de Madame de Bargeton à Angoulême reflète des traits d'un bovarysme avant la lettre, ridiculement romanesque.

Ensuite, la comparaison de diverses traductions espagnoles et anglaises d'Illusions perdues permet de vérifier, d'une part, le degré de l'attention portée par les traducteurs à l'importance capitale des discours étudiés et, d'autre part, dans quelle mesure cela a des répercussions sur la pratique de la traduction. C'est dans ce sens que l'on peut affirmer, par exemple, que les traductions anglaises tendent davantage que les espagnoles à maintenir dans la langue cible de nombreux mots français contenus dans les divers discours analysés, surtout dans les sociolectes. Cette pratique s'explique par le fait que ces mots apparus en français et répandus dans cette langue étaient attestés en anglais depuis longtemps. Quoi qu'il en soit, l'intégration de ces mots spécifiques originaux conduit plus d'une fois les traducteurs à ajouter des informations métalinguistiques qui précisent leur sens, mais qui entraînent une altération évidente du texte de départ. Quant aux idiolectes, les traductions anglaises refusent généralement d'intégrer les néologismes étonnants dont Balzac parsème les discours de ses personnages et même celui du narrateur omniscient. En revanche, ce choix est compensé au moyen de périphrases qui, même si elles restent fidèles au sens d'origine, éliminent des traits linguistiques qui sont caractéristiques des personnages. De leur côté, les traducteurs espagnols sont plus enclins à traduire les termes des sociolectes et à adapter les vocables des différents idiolectes, processus favorisé en grande partie par la similitude des aspects morphologiques de l'espagnol et du français, en somme de la proximité des deux langues de travail, et par le fait que l'espagnol a toujours eu tendance à «hispaniser» les mots étrangers, ce qui permet alors la création de textes qui respectent encore plus fidèlement le rôle original des termes sociolectaux et idiolectaux comme outils servant à la caractérisation des personnages.

Enfin, une telle analyse contrastive a permis de repérer, d'une part, de nombreux exemples de traductions littérales non justifiées, présentes dans la plupart des plus anciennes traductions espagnoles, et, d'autre part, des mutilations infligées au texte d'origine dans plusieurs d'entre elles, parfois dans le but évident d'éviter des écueils insurmontables et d'autres fois afin de censurer des allusions qui, dans le contexte d'une Espagne pas encore démocratique, pouvaient aller à l'encontre de la «bonne» morale et de la religion ${ }^{53}$.

\section{NOTES}

1. Lettre du 2 mars 1843. BAlzac, Honoré de (1832-1844/1968): Lettres à Madame Hanska, tome 2. Textes réunis, classés et annotés par Roger Pierrot. Paris: Les Éditions du Delta, 166-175.

2. On peut souligner, parmi ces marqueurs, le recours à «l'histoire parallèle», la «complémentarité sémiologique», la présence d'une "source-garante de l'information", la «forte redondance et la prévisibilité des contenus» et un «système narratif de type cyclothymique» caractérisé par de divers hauts et bas au sein de la narration. 
3. Cependant, le but final de Hamon (1973: 417), au-delà des réalismes textuel et symbolique, est l'élaboration d'une typologie du discours réaliste fondée davantage sur des manifestations discursives (système descriptif, typologie de savoirs, réseaux anaphoriques, etc.) que sur des manifestations poétiques.

4. En fait, tant l'édition de La Bibliothèque de la Pléiade (Balzac 1837-1843/1977: 1148) que la traduction de Zambrano (Balzac 1837-1843/2008: 57) ont recours à des notes explicatives pour éclaircir le sens de ces vocables.

5. Diccionario de la lengua española, Real Academia Española (2001): Entrée bruza. $22^{\mathrm{e}}$ édition. Consulté le 5 septembre 2012, <http://www.rae.es/rae.html>.

6. Trésor de la Langue française informatisé (s.d.) : Entrée prote. Consulté le 5 septembre 2012, <http:// atilf.atilf.fr/tlf.htm>.

7. Diccionario de la lengua española, Real Academia Española (2001): Entrée regente. $22^{e}$ édition. Consulté le 6 septembre 2012, <http://www.rae.es/rae.html>.

8. Oxford English Dictionary (s.d.): Entrée foreman. Consulté le 7 septembre 2012, <http://www.oed. com>.

9. Trésor de la Langue française informatisé (s.d.): Entrée savate. Consulté le 6 septembre 2012, <http:// atilf.atilf.fr/tlf.htm>.

10. Trésor de la Langue française informatisé (s.d.): Entrée savetier. Consulté le 6 septembre 2012, $<$ http://atilf.atilf.fr/tlf.htm>.

11. Diccionario de la lengua española, Real Academia Española (2001): Entrée chapucero, ra. $22^{\mathrm{e}}$ édition. Consulté le 6 septembre 2012, <http://www.rae.es/rae.html>.

12. Oxford English Dictionary (s.d.): Entrée bungler. Consulté le 7 septembre 2012, <http://www.oed. com>.

13. Trésor de la Langue française informatisé (s.d.): Entrée savate. Consulté le 6 septembre 2012, <http:// atilf.atilf.fr/tlf.htm>.

14. Trésor de la Langue française informatisé (s.d.): Entrée savate. Consulté le 6 septembre 2012, <http:// atilf.atilf.fr/tlf.htm>.

15. Oxford English Dictionary (s.d.): Entrée cobbler, n. 1. Consulté le 7 septembre 2012, <http://www. oed.com>.

16. Oxford English Dictionary (s.d.): Entrée botcher, n. 1. Consulté le 7 septembre 2012, <http://www. oed.com>.

17. Oxford English Dictionary (s.d.) : Entrée jobber, n. 2. Consulté le 7 septembre 2012, <http://www. oed.com>.

18. Oxford English Dictionary (s.d.): Entrée jobbery. Consulté le 7 septembre 2012, <http://www.oed. com>.

19. Van Rossum-Guyon (2002: 26) résume justement que «à milieu spécial langue spéciale, à monde étrange langue étrangère. D’où la nécessité pour le narrateur de traduire au fur et à mesure les termes pour le lecteur ignorant [...] Mais l'argot n'est pas seulement la langue d'un certain milieu. Sociolecte destiné à caractériser certains personnages, c'est aussi une langue secrète, une langue parallèle qui déguise la langue normale».

20. Trésor de la Langue française informatisé (s.d.): Entrée rossignol. Consulté le 6 septembre 2012, $<$ http://atilf.atilf.fr/tlf.htm>.

21. Rossignol est un terme provenant de l'argot des libraires (en fait, c'est le libraire Vidal qui parle dans cet extrait) adopté aussi par les journalistes et les critiques littéraires.

22. Il traduit rossignol par maula (Balzac 1837-1843/2007a: 261), qui signifie: «2. f. cosa inútil y despreciable» (Diccionario de la lengua española, Real Academia Española (2001): Entrée maula. $22^{\mathrm{e}}$ édition. Consulté le 5 septembre 2012, <http://www.rae.es/rae.html>).

23. Trésor de la Langue française informatisé (s.d.): Entrée rossignol. Consulté le 6 septembre 2012, $<$ http://atilf.atilf.fr/tlf.htm>.

24. Un nom dont le sens donne lieu à un autre jeu de mots avec un motif végétal: le livre de poèmes écrit par le poète nommé Chardon est intitulé précisément Les Marguerites, et les sonnets qui le composent portent des noms de fleurs diverses.

25. Oxford English Dictionary (s.d.) : Entrée nightingale. Consulté le 6 septembre 2012, <http://www. oed.com>.

26. Comme l'on peut le voir dans quelques-uns des exemples cités par l'Oxford English Dictionary: «1812 R. SOUTHEY Omniana II. 33 Walton accuses the frogs of destroying them, but I cannot persuade myself to find a true bill against these poor persecuted Dutch nightingales. 1975 D. BAGLEY Snow Tiger xi. 97 A Texas nightingale isn't a bird... It's a donkey. 1994 R. HENDRICKSON 
Happy Trails 10 Arizona nightingale. A humorous term for a braying burro or mule». Oxford English Dictionary (s.d.): Entrée nightingale. Consulté le 6 septembre 2012, <http://www.oed. com>.

27. Oxford English Dictionary (s.d.): Entrée songster. Consulté le 6 septembre 2012, <http://www.oed. com>.

28. Cependant, tant Raine (Balzac 1837-1843/2001: 57) que Marriage (Balzac 1837-1843/2009: 48) incluent le mot français chardonneret entre parenthèses: «the songster (chardonneret) of the sacred grove». Cette interférence atténue dans la langue cible l'effet connotatif du terme anglais songster.

29. Diccionario de la lengua española, Real Academia Española (2001): Entrée sonsonete. $22^{\mathrm{e}}$ édition. Consulté le 6 septembre 2012, <http://www.rae.es/rae.html>.

30. Oxford English Dictionary (s.d.): Entrée sonneteer. Consulté le 6 septembre 2012, <http://www. oed.com>.

31. Oxford English Dictionary (s.d.): Entrée sonneteer. Consulté le 6 septembre 2012, <http://www. oed.com>.

32. Prévost d’Arlincourt, Charles-Victor (1823): Ipsiboé. Paris: Béchet aîné.

33. Lewis, Matthew (1822/2004): Anaconda. Whitefish: Kessinger Publishing. En réalité, Balzac avance des événements historiques, puisque tant le conte de Lewis (1822) que le roman d'Arlincourt (1823) précèdent l'action d'Illusions perdues.

34. De StaËL, Madame (1807/1985): Corinne ou l'Italie. Coll. Folio. Paris: Gallimard.

35. Trésor de la Langue française informatisé (s.d.): Entrée tartine. Consulté le 6 septembre 2012, $<$ http://atilf.atilf.fr/tlf.htm>.

36. Trésor de la Langue française informatisé (s.d.): Entrée tartine. Consulté le 6 septembre 2012, $<$ http://atilf.atilf.fr/tlf.htm>.

37. Trésor de la Langue française informatisé (s.d.): Entrée tailler. Consulté le 7 septembre 2012, <http:// atilf.atilf.fr/tlf.htm>.

38. Trésor de la Langue française informatisé (s.d.) : Entrée tailler. Consulté le 7 septembre 2012, <http:// atilf.atilf.fr/tlf.htm>.

39. Trésor de la Langue française informatisé (s.d.): Entrée tailler. Consulté le 7 septembre 2012, <http:// atilf.atilf.fr/tlf.htm>.

40. Trésor de la Langue française informatisé (s.d.): Entrée tailler. Consulté le 7 septembre 2012, <http:// atilf.atilf.fr/tlf.htm>.

41. Trésor de la Langue française informatisé (s.d.): Entrée bavette. Consulté le 7 septembre 2012, $<$ http://atilf.atilf.fr/tlf.htm>.

42. Trésor de la Langue française informatisé (s.d.): Entrée bavette. Consulté le 7 septembre 2012, $<$ http://atilf.atilf.fr/tlf.htm>.

43. Oxford English Dictionary (s.d.): Entrée tartine. Consulté le 7 septembre 2012, <http://www.oed. com>.

44. Oxford English Dictionary (s.d.): Entrée tartine. Consulté le 7 septembre 2012, <http://www.oed. com>.

45. Diccionario de la lengua española, Real Academia Española (2001): Entrée tostada. $22^{e}$ édition. Consulté le 7 septembre 2012, <http://www.rae.es/rae.html>.

46. Diccionario de la lengua española, Real Academia Española (2001): Entrée tostón. $22^{e}$ édition. Consulté le 7 septembre 2012, <http://www.rae.es/rae.html>.

47. Diccionario de la lengua española, Real Academia Española (2001): Entrée tostón. $22^{e}$ édition. Consulté le 7 septembre 2012, <http://www.rae.es/rae.html>.

48. C'est le cas déjà commenté de saveteuse.

49. En fait, misogynie n'est attesté qu'à partir de 1812 .

50. Trésor de la Langue française informatisé (s.d.) : Entrée sultanesque. Consulté le 7 septembre 2012, $<$ http://atilf.atilf.fr/tlf.htm>.

51. Oxford English Dictionary (s.d.): Entrée sultanesque. Consulté le 7 septembre 2012, <http://www. oed.com>.

52. Un exemple similaire mais beaucoup plus étendu au long du roman est le discours du baron de Nucingen dans Splendeurs et misères des courtisanes. BalzaC, Honoré de (1838-1847/2008): Splendeurs et misères des courtisanes. Paris: Librairie Général Française.

53. C’est le cas, par exemple, de Zambrano Barragán et García (Balzac 1837-1843/1960: 806), qui éliminent le passage suivant extrait de l'entretien du pervers Vautrain, sous l'identité du faux abbé espagnol Carlos Herrera, avec Lucien Chardon: «Les uns descendent d'Abel, les autres de Caïn, dit le chanoine en terminant; moi je suis un sang mêlé: Caïn pour mes ennemis, Abel pour mes 
amis, et malheur à qui réveille Caïn!... Après tout, vous êtes Français, je suis Espagnol et, de plus, chanoine!... - Quelle nature d'Arabe!' se dit Lucien en examinant le protecteur que le ciel venait de lui envoyer». (Balzac 1837-1843/1977: 704-705).

\section{RÉFÉRENCES}

Auerbach, Erich (1983): Mimesis. La representación de la realidad en la literatura occidental. México: Fondo de Cultura Económica. (Édition originale: Mimesis. Dargstelle Wirklichkeit in der Abendländischen Literatur. Bern: A. Francke AG. Verlag, 1942).

BAKer, Mona (1992): In Other Words. A Coursebook on Translation. London/New York: Routledge.

Dagneaud, Robert (1954): Les éléments populaires dans le lexique de La Comédie humaine de Balzac. Quimper: Menez.

Damourette, Jacques et Pichon, Edouard (1971): Des mots à la pensée. Essai de grammaire de la langue française (1911-1946). Paris: Vrin.

Déruelle, Aude et Rullier-Theuret, Françoise (2003) : Illusions perdues, de Balzac. Neuilly: Atlande.

Hamon, Philippe (1973): Un discours contraint. Poétique. 16:411-445.

SAINÉAN, Lazare (1972): L’argot ancien. Genève: Slatkine reprints.

Tricás Preckler, Mercedes (2003). Manual de traducción. Francés / Castellano. Barcelona: Gedisa.

Van Rossum-Guyon, Françoise (2002): Balzac: la littérature réfléchie. Discours et autoreprésentations. Montréal: Paragraphes-Département d'Études françaises.

\section{ANNEXE}

\section{1. Éditions françaises et traductions d'Illusions perdues}

\section{1. Éditions françaises}

BAlzaC, Honoré de (1837-1843/1977): Illusions perdues. In: Roland Chollet, dir. Bibliothèque de la Pléiade. Vol. 5. Paris: Gallimard.

\subsection{Traductions espagnoles}

BALzAC, Honoré de (1837-1843/1968): Ilusiones perdidas (Traduit par Julio C. Acerete). Barcelone: Bruguera.

BalzaC, Honoré de (1837-1843/1967): Ilusiones perdidas (Traduit par Rafael Cansino). In: Balzac: Obras completas. Madrid: Aguilar.

Balzac, Honoré de (1837-1843/1964): Ilusiones perdidas (Traduit par Juan Godó). Barcelone: Lorenzana.

BAlzaC, Honoré de (1837-1843/2007a): Ilusiones perdidas (Traduit par José R. Monreal). Barcelone: DeBolsillo-Random House Mondadori.

BALZAC, Honoré de (1837-1843/1970) : Ilusiones perdidas (Traduit par José RiberA). Barcelone: Petronio.

BALZAC, Honoré de (1837-1843/1960) : Ilusiones perdidas (Traduit par José Zambrano BARRAGán et Joaquín García). Madrid: Edaf.

Balzac, Honoré de (1837-1843/2008): Ilusiones perdidas (Traduit par Pablo Zambrano). Barcelona: Rba-Gredos.

\subsection{Traductions anglaises}

BalzaC, Honoré de (1837-1843/2004) : Lost Illusions (Traduit par Herbert J. HunT, 1971). London: Penguin.

BalzaC, Honoré de (1837-1843/2009): Lost Illusions (Traduit par Ellen Marriage). Milton Keynes: Dodo Press. 
646 MeTA, LVII, 3, 2012

Balzac, Honoré de (1837-1843/2007b) : Lost Illusions (Traduit par Katherine Prescott, 1893). New York: Barnes \& Noble.

Balzac, Honoré de (1837-1843/2001): Lost Illusions (Traduit par Kathleen Raine, 1951). New York: The Modern Library. 